\title{
PROBLÈMES DIGESTIFS
}

\section{Hémorragies digestives}

Il ne faut pas hésiter à admettre pour surveillance à l'USI un patient présentant une hémorragie digestive, même relativement bénigne, dans la mesure où l'évolution de l'hémorragie est difficile à prévoir.

La réanimation initiale comprend:

- protection des voies aériennes et oxygénothérapie généreuse. L'intubation endotrachéale peut être souhaitable avant le traitement endoscopique;

- placement d'au moins une bonne voie d'entrée (deux si possible); un cathéter veineux central est préférable pour faciliter un remplissage vasculaire rapide, tout en contrôlant la pression veineuse centrale; transfusions sanguines et administration de FFP en cas de troubles de la coagulation. En cas d'hémorragie importante, il ne faut toutefois pas nécessairement maintenir la normotension, qui risque de faciliter le saignement;

- placement d'un cathéter artériel permettant la surveillance de la pression artérielle et facilitant les prélèvements sanguins;

- placement éventuel d'une sonde gastrique. La mise en place d'une large sonde (même chez les patients dont la source de saignement pourrait être basse) permet de reconnaître quasi tout saignement œso-gastro-duodénal (un saignement duodénal sans reflux gastrique peut survenir), de surveiller le saignement, d'évacuer le sang et d'éviter la distension gastrique. Le lavage gastrique à l'eau glacée n'a pas de place thérapeutique. Toutefois, le placement d'une sonde gastrique est évité en cas de pathologie œsophagienne ou d'ulcération secondaire à la sonde gastrique déjà en place... En cas de cirrhose connue, la prise en charge est cependant plus spécifique (voir plus bas);

- administration d'agents vasopresseurs en cas d'hypotension sévère. 


\section{Examens complémentaires}

\section{Examens endoscopiques}

Eso-gastro-duodénoscopie: examen de choix pour les hémorragies hautes ou basses, à pratiquer d'urgence en cas d'hémorragie importante, continue ou récurrente (tableau I); L'examen se déroule sans anesthésie pharyngée et avec un minimum de sédation (risque d'inhalation). L'injection IV lente de $250 \mathrm{mg}$ d'érythromycine 20 minutes avant l'endoscopie peut faciliter l'examen endoscopique par son effet prokinétique.

Colonoscopies pour les hémorragies basses en cas d'œso-gastro-duodénoscopie négative.

Tableau I - Classification de Forrest des hémorragies digestives.

\begin{tabular}{|l|c|c|}
\hline Saignement actif (Forrest I) & Mortalité & Récidives \\
FIa: jet artériel (« spurting») & $40 \%$ & $80 \%$ \\
FIb: suintement (« oozing») & $20 \%$ & $30 \%$ \\
Saignement récent (Forrest II) & & \\
FIIa: vaisseau visible & $15 \%$ & $40 \%$ \\
FIIb: caillot & $0 \%$ & $20 \%$ \\
FIIc: tache noire & & $10 \%$ \\
Pas de saignement (Forrest III) & & \\
\hline
\end{tabular}

\section{Examens angiographiques}

En cas d'hémorragie importante, surtout si l'endoscopie ne peut préciser ou contrôler la source du saignement. L'examen angiographique permet non seulement d'identifier la source du saignement, mais aussi le cas échéant un traitement local par l'embolisation sélective de l'artère incriminée et éventuellement l'administration locale de vasoconstricteurs (supériorité contestée par rapport à l'administration systémique). Il convient toujours dans ces cas de discuter avec les chirurgiens de la meilleure attitude à adopter en fonction des comorbidités du patient.

Le saignement doit être d'au moins $1 \mathrm{~mL} / \mathrm{min}$ (60 mL/heure) pour que l'examen soit contributif. 


\section{Traitement médical}

\section{Ulcère gastroduodénal}

\section{Traitement:}

- contrôle endoscopique du saignement (par clip, argon ou électrocoagulation bipolaire) avec consolidation éventuelle (en principe le lendemain);

- traitement antiacide par inhibiteurs de la pompe à protons (en cas de lésion visualisée): oméprazole IV $80 \mathrm{mg}$ en bolus puis administration continue de $200 \mathrm{mg}$ /jour pendant $72 \mathrm{~h}$ Les anti-H2, comme la ranitidine, sont moins efficaces, et ne sont plus utilisés dans ce contexte;

- administration éventuelle de somatostatine pour réduire le débit sanguin local (indication pas clairement établie).

\section{Varices œsophagiennes}

Les varices œsophagiennes sont la conséquence de l'hypertension portale. Le gradient de pression veineuse hépatique est typiquement supérieur à $10 \mathrm{mmHg}$ pour que les varices se forment et supérieur à $12 \mathrm{mmHg}$ pour qu'elles se rompent.

L'hypertension portale sévère entraîne des anastomoses systémiques notamment dans la région inférieure de l'œsophage. La grande majorité des patients cirrhotiques a des varices œesophagiennes et 25 à $40 \%$ des cirrhotiques présentent à un moment ou un autre une hémorragie de varices. Le saignement de varices œsophagiennes est responsable d'un tiers des décès des patients cirrhotiques. Le diagnostic se fait par endoscopie; même si différents systèmes de gradation des varices ont été proposés, il est recommandé de s'en tenir à une classification très simple (tableau II). Les varices peuvent également être gastriques (20-40\%) et plus rarement ectopiques (antrales, duodénales...).

Tableau II - Classification des varices œsophagiennes.

Grade 1: rubans veineux de petite taille, s'affaissant à l'insufflation.

Grade 2: varices non aplaties par l'insufflation et séparées par de la muqueuse saine.

Grade 3: varices de grande taille, faisant protrusion dans la lumière et pratiquement confluentes.

\section{Traitement du saignement sur varices œsophagiennes}

Maintien des fonctions vitales (règle du VIP): voir plus haut.

Dans ce cas précis, il ne faut pas chercher à restaurer un taux d'hémoglobine de plus de $8 \mathrm{~g} / \mathrm{dL}$, car les transfusions généreuses favorisent la persistance ou la récidive 
du saignement. L’administration de FFP n'est généralement pas indiquée car elle n'a aucune valeur hémostatique démontrée.

Traitement médicamenteux:

- la vasopressine a longtemps été recommandée $(1 \mathrm{U} / \mathrm{min}$ pendant environ 20 minutes, puis 0,1 à $0,5 \mathrm{U} / \mathrm{min}$ ) pour ses effets vasoconstricteurs sur la circulation splanchnique, mais les effets vasoconstricteurs myocardiques, mésentériques et périphériques représentent un problème sérieux; l'addition de nitroglycérine a été proposée pour pallier ces effets vasoconstricteurs. La terlipressine peut être administrée à raison de 1 à $2 \mathrm{mg}$ (selon le poids) toutes les 4 heures pendant 5 jours;

- la somatostatine est au moins aussi efficace que la vasopressine, mais entraîne moins d'effets secondaires, si bien qu'elle l'a remplacée dans cette indication. La somatostatine diminue l'hyperhémie et réduit la pression portale par l'intermédiaire de peptides vasoactifs (substance $\mathrm{P}$, glucagon). Les effets secondaires sont l'hyperglycémie et les douleurs crampoïdes abdominales. On commence par un bolus de $250 \mathrm{mcg}$, suivi d'une perfusion de $250 \mathrm{mcg} / \mathrm{heure}$, soit $6 \mathrm{mg}$ par 24 heures. On peut éventuellement ajouter des bolus supplémentaires de $250 \mathrm{mcg}$.

L'octréotide (Sandostatine ${ }^{\circledR}$ ) est un analogue de synthèse de la somatostatine, qui a une demi-vie plus longue (voir plus loin).

Éviter les médicaments néphrotoxiques il convient de bannir toute forme de néphrotoxicité iatrogène (aminoglycosides, AINS...), la fonction rénale du malade cirrhotique étant déjà altérée de par la maladie sous-jacente.

\section{Traitement endoscopique}

L'endoscopie est toujours nécessaire, car une cause autre que les varices est retrouvée dans $20 \%$ des cas des hémorragies digestives chez les cirrhotiques (en majorité des ulcères). En revanche, la gastropathie d'hypertension portale n'est pas une cause d'hémorragie aiguë.

Trois procédures endoscopiques sont possibles:

- la sclérothérapie: elle est facile à réaliser, sous contrôle visuel direct et bon marché. L'injection du produit sclérosant arrête le saignement dans 80-90\% des cas, mais présente un taux de complications de $20 \%$, sous forme de perforations, ulcérations et de rétrécissement;

- la ligature des varices: les résultats sont meilleurs, mais elle peut être difficile à réaliser au cours de l'hémorragie active; elle nécessite aussi une plus grande expérience endoscopique; les complications sont un peu moins fréquentes et consistent surtout en ulcérations muqueuses;

- l'obturation à la colle est la plus efficace (> $95 \%$ d'hémostase), mais elle nécessite un opérateur entraîné. Ce polymère (Histoacryl ${ }^{\circledR}$ ) durcit en présence de sang. Elle est utilisée surtout dans les hémorragies massives ou en cas de varices gastriques et ectopiques. La complication principale (2\%) est l'embolisation de colle vers le système porte ou pulmonaire, ou même l'embolisation artérielle s'il existe une communication interauriculaire.

\section{Tamponnade}

Compression des varices par différents types de sondes placées sous traction:

- sonde de Blakemore: ballon gastrique de $300 \mathrm{~mL}$, ballonnet œsophagien de 45- 
$60 \mathrm{~mL}$ et aspiration gastrique; il est recommandé aujourd'hui de ne plus gonfler le ballonnet œesophagien;

- sonde de Linton: ballon gastrique de $600 \mathrm{~mL}$ d'air et aspiration gastrique et œsophagienne.

Ces sondes, dont le placement exige une certaine habileté, ne sont efficaces que dans environ $50 \%$ des cas, et les récidives sont fréquentes lors de leur enlèvement. Les complications consistent en l'inhalation de contenu gastrique et la nécrose et même la rupture œsophagienne. Ces manœuvres de tamponnade ne sont plus utilisées qu'exceptionnellement.

TIPS (tranjugular intrahepatic portosystemic shunt)

Il s'agit de l'équivalent d'un shunt chirurgical porto-cave latéro-latéral, mais placé en intrahépatique par voie veineuse, donc sans les inconvénients de la laparotomie. Il peut être proposé en cas d'hémorragie sur varices non controlable endoscopiquement.

Antibiothérapie prophylactique et traitement du sepsis

Un sepsis sous-jacent peut être un facteur favorisant le saignement des varices et la récidive hémorragique. Il doit donc être recherché activement et traité empiriquement. En plus des hémocultures, la présence d'ascite impose un prélèvement du liquide pour culture. Une antibiothérapie empirique de 48-72 heures est recommandée après les manœuvres endoscopiques, et est réévaluée lorsque les examens bactériologiques sont disponibles. Même en l'absence d'infection sous-jacente, l'administration prophylactique d'antibiotiques réduit la mortalité chez ces patients. Chez le malade venant de l'extérieur, l'amoxycilline-clavulanate (Augmentin ${ }^{\circledR}$ ) suffit dans cette indication.

Prévention de l'encéphalopathie hépatique

Administration de lactulose, lavement éventuel

\section{Traitement au long cours}

Le risque de récidive de saignement est très important (30\%) au cours des mois qui suivent, si bien que le traitement doit être complété par une prophylaxie secondaire:

- séances répétées de ligature de varices (ou de sclérothérapie);

- administration de bêta-bloquants non sélectifs (bêta-1 pour diminuer le débit cardiaque; bêta-2 pour diminuer sélectivement le débit splanchnique), éventuellement en combinaison avec des dérivés nitrés;

- TIPS («transjugular intrahepatic portosystemic shunt») comme procédure efficace pour diminuer l'hypertension portale; toutefois l'encéphalopathie peut survenir dans $25 \%$ des cas chez ces malades (surtout si le score de Child est élevé), si bien qu'il n'est pas indiqué en première intention.

\section{Prévention des ulcères de stress}

Les pathologies les plus fréquentes sont la gastrite hémorragique et les ulcérations gastriques aiguës, toutes deux liées au stress, et peuvent survenir chez tout patient 
malade grave (traumatisme, choc, sepsis, brûlures [ulcère de Curling], insuffisance rénale, insuffisance respiratoire...). Les ulcérations muqueuses sont superficielles et concernent plus fréquemment le fundus que l'antre. Ces ulcérations sont fréquentes chez le malade grave, mais heureusement saignent rarement. Leur saignement est d'ailleurs de très mauvais pronostic (en général MOF déjà avancé).

\section{Traitement préventif}

La prévention systématique des hémorragies digestives n'est pas nécessaire chez tous les malades d'USI.

Les principaux facteurs de risque sont:

- l'insuffisance respiratoire nécessitant la ventilation mécanique;

- les altérations de coagulation;

- le sepsis sévère et le MOF;

- le trauma crânien;

- les brûlures sévères;

- les médications augmentant le risque d'ulcères de stress: corticostéroïdes, AINS...

Élévation du $\mathrm{pH}$ gastrique par anti-H2: son efficacité est établie. L'élévation du $\mathrm{pH}$ gastrique peut favoriser le développement bactérien dans l'estomac et par là favoriser le développement d'infections pulmonaires nosocomiales. Toutefois, le réel impact de cette complication est relativement limité.

« Tapissage » de la muqueuse gastrique par sucralfate: bien qu'il n'augmente pas le $\mathrm{pH}$ gastrique, le sucralfate se lie aux sels biliaires et à la pepsine, améliore la qualité de la barrière muqueuse et augmente ses capacités de neutralisation. Le sucralfate n'a pas d'interaction médicamenteuse notable. Son efficacité semble toutefois moindre que celle des anti-H2.

Inhibiteurs de la pompe à protons: ce sont les seules molécules capables d'inhiber totalement la sécrétion acide; (même si leur efficacité dans la prévention des ulcères de stress n'a pas été démontrée). Le coût est la limitation principale à leur administration routinière.

Antiacides: ils sont moins efficaces que les anti-H2 et présentent plus d'effets secondaires.

Nutrition entérale précoce.

De manière générale, une réanimation rapide et efficace!

\section{Abdomen aigu}

\section{Anamnèse}

Il convient de préciser notamment:

- le moment d'installation de la douleur et la rapidité de son développement;

- les facteurs aggravant la douleur (mouvements, toux, respiration, nourriture) et 
soulageant (repos, position particulière, vomissements, nourriture, médications...);

- l'endroit le plus sensible, et l'irradiation;

- la présence de nausées et de vomissments;

- la présence de constipation ou de diarrhées - de méléna;

- la perte d'appétit et de poids éventuelles.

Le type de douleur peut aussi orienter le diagnostic:

- brutale: anévrisme de l'aorte abdominale, rupture de viscère, grossesse extrautérine...;

- intense, d'aggravation rapide: cholécystite, pancréatite, infarctus mésentérique;

- progressive, stable: appendicite, diverticulite, inflammation pelvienne, hernie, obstruction intestinale;

- constante: péritonite, ischémie intestinale;

- intermittente: obstruction intestinale, obstruction biliaire, urétérale ou de trompe de Fallope (douleurs intenses).

L'examen clinique doit comprendre:

- l'observation: recherche de cicatrice, reconnaissance de respiration abdominale;

- la palpation: rigidité, distension, défense, rebond, masse;

- les touchers rectal et vaginal;

- la percussion;

- la recherche de péristaltisme.

Ischémie mésentérique aiguë

Elle représente une cause fréquente d'abdomen aigu, soit par embolie (60\%), par thrombose artérielle $(30 \%)$ ou thrombose veineuse $(10 \%)$. Le bas débit et l'hypovolémie sont des facteurs favorisants.

Le diagnostic est posé essentiellement par CT-scan.

\section{Fistules digestives}

Il s'agit de complications postopératoires dans $80 \%$ des cas (cancer, pathologies inflammatoires...).

Le traitement est initialement conservateur, en mettant le tube digestif au repos (avec alimentation parentérale) et en corrigeant éventuellement les désordres hydroélectrolytiques. Un traitement chirurgical est considéré ultérieurement, mais généralement réservé aux cas compliqués. Une solution endoscopique peut aussi parfois être proposée.

La somatostatine peut être utile, par ses effets inhibiteurs d'hormones pituitaires et digestives, dont l'hormone de croissance, la TSH, le glucagon, la cholécystokinine, le VIP (vasoactive intestinal peptide) et la gastrine. Elle inhibe la sécrétion acide de l'estomac et diminue le transit gastro-intestinal et l'activité de la vésicule.

La somatostatine est un peptide (14 acides aminés) qui a une demi-vie de quelques minutes, si bien qu'elle doit être administrée en perfusion continue. Elle présente des effets vasopresseurs, pouvant entraîner une élévation de pression artérielle et un ralentissement de la fréquence cardiaque. Elle peut aussi entraîner de l'hyperglycémie. 
L'octréotide (Sandostatine ${ }^{\circledR}$ ) est un analogue de synthèse de la somatostatine, qui a une demi-vie de quelques heures, permettant l'administration par voie sous-cutanée trois fois par jour (100 mcg par injection). Une forme prolongée (Sandostatine ${ }^{\circledR}$ LAR) est même disponible pour les administrations de longue durée. Une seule injection intramusculaire permet la libération de somatostatine pendant plusieurs semaines.

Ces substances sont aussi utiles dans les diarrhées rebelles.

\section{lléus}

En règle générale, l'iléus nécessite l'arrêt de la nutrition et l'aspiration du contenu gastrique. Toutefois, en l'absence de pathologie organique, l'administration prudente d'alimentation entérale peut aider à rétablir le transit.

L'iléus postopératoire persistant peut être dû à différents facteurs, parmi lesquels:

-l'influence persistante des agents morphiniques;

-l'inflammation;

-l'influence de différents facteurs sympathique set hormonaux endogènes.

Pour contrebalancer les effets anticholinergiques de l'iléus paralytique, on peut administrer de la physostigmine, en tant qu'inhibiteur réversible de l'acétylcholinesterase. La dose de néostigmine est de $2 \mathrm{mg}$ par voie IV (éventuellement souscutanée), à répéter régulièrement, même toutes les 2 heures. Certains l'administrent même sous forme de perfusion intraveineuse, à raison de 0,4 à $0,8 \mathrm{mg} /$ heure. Les effets secondaire sont de type sympathicomimétique (hypertension et tachycardie). Le métoclopramide (Primperan ${ }^{\circledR}$ ) et l'érythomycine sont inefficaces dans ces conditions.

\section{Syndrome d'Ogilvie}

Il s'agit d'une pseudo-obstruction du côlon, due probablement à une stimulation parasympathique intense.

Le diagnostic est à la fois clinique (distension colique) et radiologique (dilatation du côlon droit surtout).

Le traitement est essentiellement conservateur: correction des troubles hydroélectrolytiques, mise au repos du tube digestif, sonde rectale (s'assurer qu'il ne s'agit pas d'un fécalome), lavement éventuel. Lorsque le diamètre du cæcum est supérieur à $10 \mathrm{~cm}$ le risque de perforation est très élevé et le traitement devient une nécessité absolue.

L'administration de néostigmine $(2 \mathrm{mg} \mathrm{IV})$, à répéter si nécessaire, permet d'obtenir un flatus dans $80 \%$ des cas (réduction du diamètre et évacuation de l'aérocolie). Par contre, l'administration de cisapride n'est pas très efficace et potentiellement dangereuse en raison du risque d'arythmie. Pour les $20 \%$ restants, la colonoscopie de désinsufflation est indiquée. Le recours à la chirurgie est exceptionnel.

\section{Syndrome compartimental abdominal}

"L'abdominal compartment syndrome » est un tableau décrit relativement récemment, et pourtant important à reconnaître (tableau III). L'augmentation de 
pression peut être due à de l'œdème, à la présence d'hématomes ou d'autres collections. Le problème peut être intra- ou rétropéritonéal.

Répercussions hémodynamiques:

- augmentation des pressions intrathoraciques (PVC, PAPO ...);

- diminution du débit cardiaque (voir fig. 1);

- hypoxémie;

- diminution de la diurèse secondaire à l'altération de perfusion rénale;

- diminution de la perfusion splanchnique, par diminution du débit cardiaque et augmentation des résistances vasculaires;

- élévation de la pression intracrânienne, par diminution du retour veineux du cerveau.

Tableau III - Principales situations cliniques exposant au risque de syndrome compartimental abdominal.

Laparotomie récente avec hémorragies, œdème, etc. (traumatisme abdominal, chirurgie de l'aorte abdominale, transplantation hépatique...).

Pancréatite aiguë sévère.

Hémorragie rétropéritonéale.

Néoplasie avancée.

Pneumopéritoine.

Ascite sévère.

Brûlures de la paroi abdominale.

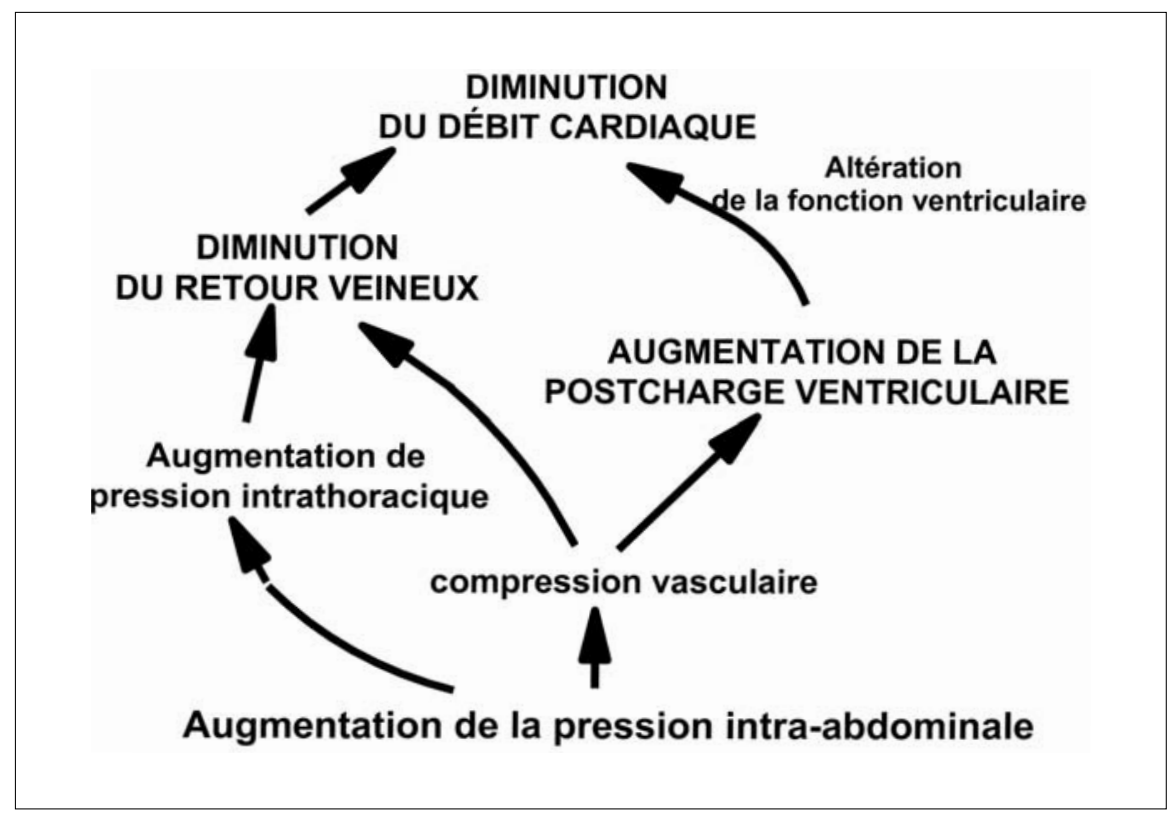

Fig. 1 - Diminution du débit cardiaque. 
Le diagnostic est posé par la mesure de la pression intravésicale, en introduisant 50 à $100 \mathrm{~mL}$ de solution salée dans la sonde vésicale, en clampant la sonde et en mesurant la pression au moyen d'un transducteur standard de pression intravasculaire.

La pression n'excède normalement pas $10 \mathrm{mmHg}$. Une pression intravésicale supérieure à $15 \mathrm{mmHg}$ peut entraîner des conséquences (tableau IV), qui deviennent sévère lorsqu'elle dépasse $30 \mathrm{mmHg}$.

Un "fluid challenge » doit être considéré d'abord. Un traitement chirurgical peut être nécessaire pour assurer la décompression abdominale, surtout lorsque la pression dépasse $25 \mathrm{mmHg}$ (il faut parfois laisser l'abdomen ouvert).

Tableau IV - Attitudes en fonction du niveau de pression intra-abdominale.

$\begin{array}{ll}\text { Pression intravésicale } & \text { Attitude } \\ -10-15 \mathrm{mmHg}: & \begin{array}{l}\text { assurer la normovolémie - considérer un } \\ \text { «fluid challenge» }\end{array} \\ -16-25 \mathrm{mmHg}: & \text { mantenir une hypervolémie } \\ ->25 \mathrm{mmHg}: & \text { décompression/ré-exploration chirurgicale }\end{array}$

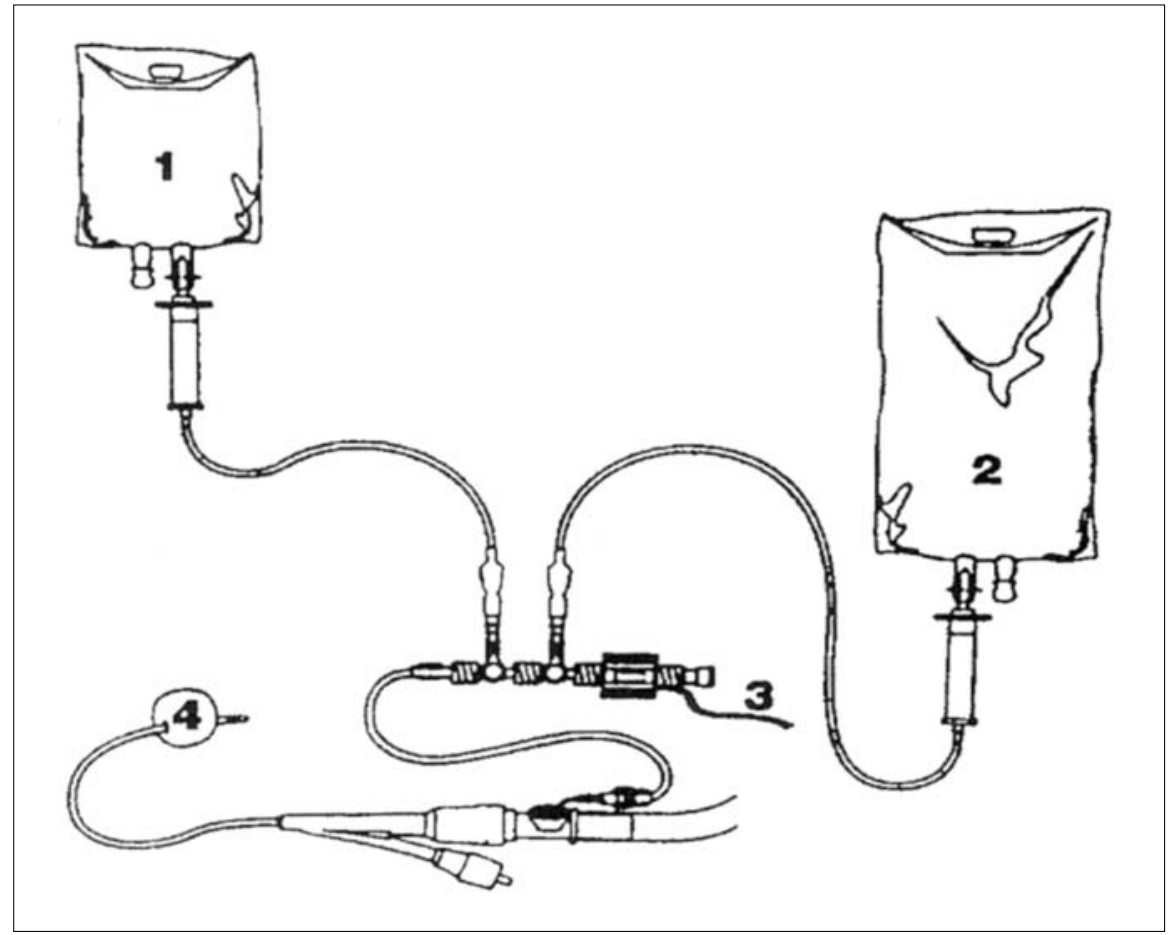

Fig. 2 - Représentation schématique de la mesure de pression intravésicale. 1) solution salée stérile. 2) tubulure. 3) transducteur de pression. 4) cathéter vésical avec ballon gonflé et aiguille. 


\section{Cirrhose}

La cirrhose est définie comme un état pathologique diffus caractérisé par le développement de fibrose associé à la transformation nodulaire du foie. On classifie ainsi sur le plan pathologique la cirrhose micronodulaire et macronodulaire, selon que ces nodules excédent ou non $3 \mathrm{~mm}$ de diamètre.

Différentes causes de cirrhose peuvent être considérées (tableau V).

Tableau V - Causes principales de cirrhose.

Toxiques: alcool

Infectieuses: hépatites B et C, schistosomiase

Médicamenteuses: méthotrexate, INH

Auto-immunitaires: cirrhose biliaire primitive, hépatite auto-immunitaire, cholangite sclérosante

Métabolique: maladie de Wilson, hémochromatose, déficience en alphaantitrypsine

Obstruction biliaire (cirrhose biliaire secondaire)

Vasculaire: syndrome de Budd-Chiari, maladie veino-occlusive

Cryptogénique

\section{Diagnostic}

Échographie, surtout avec Doppler couleur, qui permet de reconnaitre la présence d'hypertension portale; permet aussi d'exclure une pathologie des voies biliaires.

CT-scan: permet aussi de faire certains diagnostics l'hémochromatose (augmentation de densité suite aux dépôts de fer) ou des complications comme l'hépatome.

Résonance magnétique nucléaire: peut aider à caractériser les lésions focales, comme les métastases.

Biopsie percutanée: risques limités (hémorragie, péritonite biliaire, perforation d'autres viscères) si le PT est acceptable; la biopsie par laparoscopie a l'avantage de visualiser l'intervention. La biopsie par voie transjugulaire est préférée chez les malades qui ont des altérations plus sévères de la coagulation (moins de risque de saignement intraabdominal) et permet en plus l'évaluation du gradient portosystémique (évaluation indirecte de la pression porte). 


\section{Complications}

\section{Ascite}

Sa formation est due à plusieurs mécanismes:

- vasodilatation périphérique, menant à l'hypovolémie relative, responsable de l'activation du système rénine-angiotensine-aldostérone, augmentation de l'activité sympathique et augmentation de la production d'ADH;

- hypertension dans les sinusoïdes, entraînant l'extravasation liquidienne;

- diminution de l'élimination d'hormones (aldostérone en particulier);

- diminution de la pression oncotique du plasma.

Traitement:

- mobilisation du liquide d'ascite par une balance sodée négative: régime pauvre en sel, spironolactone, furosémide ;

- ponction d'ascite sous perfusion d'albumine.

\section{Encéphalopathie}

Il y a trois théories qui rendent compte de l'encéphalopathie hépatique.

L'ammoniaque: une série de neurotoxines, dont l'ammoniaque, des mercaptans et des acides gras à chaîne courte, traversent la membrane hémato-méningée; de plus, le métabolisme de l'ammoniaque peut consommer le glutamate disponible dans le cerveau; cette théorie est à la base de la décontamination digestive.

Les faux transmetteurs: les acides aminés aromatiques, dont les taux sont augmentés, augmentent l'inhibition neuronale; cette théorie est à la base de l'administration d'acides aminés branchés.

La stimulation des récepteurs à l'acide gamma-aminobutyrique (GABA): le GABA, dont les taux sont augmentés dans la cirrhose, est un important inhibiteur de la neurotransmission, lié de près aux récepteurs aux benzodiazépines; cette théorie est à la base de l'administration de flumazénil, avec des résultats toutefois peu concluants.

\section{Causes}

Les principaux facteurs pouvant précipiter l'encéphalopathie hépatique sont:

-hémorragie digestive;

-infection (dont la péritonite);

-troubles métaboliques: hypokaliémie, alcalose métabolique;

-hypovolémie, déshydratation (diurétiques, diarrhées);

-excès de calmants; benzodiazépines...:

-anémie, hypoxémie;

-facteurs favorisants: shunts porto-sytémiques, développement d'hépatome. 
Les causes peuvent être multiples. Par exemple, une hémorragie digestive est souvent compliquée de sepsis, une hyponatrémie peut être surajoutée... Une ponction d'ascite doit être considérée pour exclure une péritonite spontanée, dont la symptomatologie est parfois frustre.

\section{Diagnostic}

Haleine d'insuffisance hépatique.

Taux d'ammoniaque: le taux d'ammoniaque est typiquement élevé dans l'encéphalopathie hépatique $(100 \mathrm{mcg} / \mathrm{dL})$, mais il n'est que faiblement corrélé à la sévérité de l'encéphalopathie.

Un test de reconnaissance de structures (test de l'étoile ou connections de nombres) peut aider à reconnaître une encéphalopathie débutante.

EEG: ondes triphasiques (5 cycles/secondes) - peut montrer un tracé de type delta en cas de coma profond (grade 4).

\section{Sévérité}

(Voir tableau VII).

Tableau VII - Niveaux d'encéphalopathie hépatique.

\begin{tabular}{|l|l|l|l|}
\hline Grade & Comportement, conscience & Signes neurologiques & Signes EEG \\
\hline 1 & $\begin{array}{l}\text { pertes d'attention, } \\
\text { désorientation } \\
\text { légère, agitation, irritabilité, } \\
\text { insomnie, somnolence }\end{array}$ & $\begin{array}{l}\text { tremblements, } \\
\text { incoordination }\end{array}$ & ondes triphasiques \\
\hline 2 & $\begin{array}{l}\text { troubles du comportement, } \\
\text { léthargie, confusion }\end{array}$ & $\begin{array}{l}\text { hyperréflexie } \\
\text { asterixis, dysarthrie }\end{array}$ & ondes triphasiques \\
\hline 3 & somnolence, désorientation & $\begin{array}{l}\text { rigidité, hyperréflexie, signe } \\
\text { de Babinski }\end{array}$ & ondes triphasiques \\
\hline 4 & coma & décérébration & ondes delta \\
\hline
\end{tabular}

\section{Traitement}

\section{Altérations de la flore intestinale}

L'approche consiste à diminuer la production d'ammoniaque par des lavements et l'administration de disaccharides non résorbés comme le lactulose ou le lactulol. 
Ce dernier a deux mécanismes d'action:

- action cathartique;

- diminution du $\mathrm{pH}$ colique, favorisant le développement de bactéries non productrices d'uréase par rapport à celles productrices d'uréase. De plus, l'acidification augmente le déplacement de l'ammoniaque du sang vers la lumière digestive.

Les doses sont initialement de $6 \times 1$ cuiller à soupe par jour (30 à 60 grammes), à adapter pour obtenir 3 à 4 selles semi-liquides par jour. Si le tube digestif n'est pas fonctionnel, le lactulose peut être administré par lavements (1 à 3 litres de lactulose $20 \%$ par jour).

On peut aussi administrer des antibiotiques comme la néomycine (6 g/jour), qui agit en éliminant de préférence les bactéries productrices d'uréase. Il existe malgré tout un faible risque de résorption intestinale, avec risque d'effets ototoxiques et néphrotoxiques. L'administration de néomycine doit être évitée chez l'insuffisant rénal. L'addition de néomycine au lactulose n'est cependant pas très utile, et doit être considérée seulement en cas de réponse insatisfaisante au lactulose seul.

L'administration de bactéries ne produisant pas d'uréase (Lactobacillus acidophilus) est d'efficacité plus douteuse.

\section{Autres interventions à visée métabolique}

La restriction protéique n'a pas de place chez le malade de soins intensifs. Tout au plus, le remplacement de protéines animales par des protéines végétales peut être utile, notamment par le fait que l'augmentation des apports en fibres peut accélérer le transit intestinal et diminuer le $\mathrm{pH}$ du côlon.

Des suppléments en aspartate et en ornithine ou en benzoate de sodium peuvent accélérer le métabolisme de l'ammoniaque. Le zinc est essentiel à plusieurs processus enzymatiques, et des suppléments de zinc sont nécessaires chez ceux qui en sont déficients.

Puisque l'administration de solutions riches en acides aminés aromatiques est susceptible d'accroître le degré d'encéphalopathie, le support nutritif peut favoriser des formules enrichies en acides aminés branchés (Amino-hepa, Fresubin-hepa), avec toutefois des résultats mitigés.

L'encéphalopathie peut s'aggraver rapidement, en particulier au cours de l'insuffisance hépatique aiguë et se compliquer alors d'œè̀me cérébral.

Attitude:

- maintenir la tête légèrement surélevée à $30^{\circ}$ (une plus grande élévation risque de diminuer la perfusion cérébrale);

- éviter l'hyperventilation (qui risque d'entraîner une ischémie cérébrale) chez le malade ventilé mécaniquement et en encéphalopathie sévère;

Dans les cas les plus graves, certains mesurent la pression intracrânienne (cathéter extradural), avec le but de la maintenir en dessous de $30 \mathrm{mmHg}$;

- mannitol (solution à $20 \%$ ): 0,5 mg/kg à $1 \mathrm{~g} / \mathrm{kg}$ en $10 \mathrm{~min}$, en s'assurant que l'osmolarité plasmatique n'excède pas $320 \mathrm{mOsm}$;

- éventuellement coma barbiturique (thiopental 3-5 mg/kg - voir chapitre Soins intensifs neurologiques). 


\section{Insuffisance hépatique aiguë}

\section{Classification}

On parle d'insuffisance hépatique aiguë en présence d'altération des tests hépatiques et de la coagulation sans maladie hépatique chronique sous-jacente. Celle-ci est:

- sévère: lorsque le PTT (ou mieux le facteur 5) est $<$ à $50 \%$ en l'absence d'encéphalopathie;

- fulminante: lorsque l'encéphalopathie survient endéans les 15 jours suivant le début de l'ictère ;

- subfulminante: lorsque l'encéphalopathie survient plus de 15 jours après le début de l'ictère.

On parle d'insuffisance hépatique chronique lorsqu'elle persiste plus de 6 mois.

\section{Causes les plus fréquentes}

L'insuffisance hépatique aiguë est généralement due à une nécrose hépato-cellulaire étendue.

- hépatite virale (2/3 des cas, HAV et HBS) ;

- toxiques: paracétamol (acétaminophen des Américains - voir section toxicologique), ammanite phalloïde, hydrocarbures, ecstasy, herbes chinoises...

- autres: causes vasculaires, maladie de Wilson, stéatose aiguë de la grossesse, syndrome de Reye...

$N B$ : l'intoxication au paracétamol survient en général après l'ingestion de plus de $12 \mathrm{~g}$ de la substance (cette dose léthale, peut être largement inférieure si il existe un éthylisme sous-jacent qui diminue les réserves en glutathion du foie).

\section{Démarche diagnostique et thérapeutique}

\section{Diagnostic}

Tous les moyens doivent être rapidement mis en ouvre pour obtenir un diagnostic étiologique:

- établir l'existence d'une hépatopathie chronique sous-jacente éventuelle: examen physique, échographie hépato-splénique, gastroscopie et biopsie hépatique transjugulaire si nécessaire;

- prélèvements sanguins ciblés pour le diagnostic et pour transplantabilité (virus IgM HAV, IgM anti-HBc, herpès, CMV, HCV, HIV, gamma-globulines, FAN, antiLKM, anti-mmLisses, screening toxicologique, groupe sanguin...);

- anamnèse soignée du patient et de ses proches (contacts viraux, voyages, habitudes sexuelles, medicaments, tisanes, syndrome grippal, problèmes gynécologiques...). 
Certains éléments biologiques peuvent déjà mettre sur la piste, comme par exemple:

- transaminases $>7000=$ paracétamol ou hépatite hypoxique ou herpès;

- hypergamma-globulinémie = hépatite auto-immune ;

- histoire familiale, consanguinité, jeune âge = Wilson...

Traitement étiologique; il doit être entrepris rapidement:

intoxication au paracétamol (et aussi parfois dans d'autres cas) : $\mathrm{N}$-acétylcystéine (augmente les réserves en glutathion) : $300 \mathrm{mg} / \mathrm{kg}$ en 20 heures;

- pathologie auto-immunitaire: corticoïdes;

- herpès: acyclovir;

- maladie de Wilson: D-Pen...

\section{Évaluation pronostique et décisionnelle}

Il convient de classer le malade dès son admission: insuffisance hépatique aiguë " simple », sévère, fulminante ou subfulminante (seule l'insuffisance hépatique aiguë « simple » peut être gérée en dehors d'un centre de transplantation hépatique).

Il faut alors arrêter tout traitement non étiologique (et en tenir compte pour l'évaluation clinique du malade: ex BZP) et ne plus rien donner (sauf traitement étiologique). Il faut en particulier proscrire l'administration de substances visant à améliorer la coagulation (comme le FFP) ou prévenir l'encéphalopathie (comme le lactulose), puisque l'indication d'une transplantation sera basée sur les tests de coagulation et le degré d'encéphalopathie.

Support extracorporel:

- système MARS ( "molecular absorbent recirculating system ») ou Prometheus: il s'agit d'une dialyse utilisant un dialysat riche en albumine, pour faciliter l'élimination de toxines liées à l'albumine comme la bilirubine, les acides aminés aromatiques et des substances hydrosolubles. L'efficacité n'est pas encore définitivement prouvée ;

- expérimental: foie artificiel extracorporel.

\section{Évaluer l'indication de transplantation (tableau VIII)}

Si la décision de transplantation est prise, tous les moyens doivent être mis en œuvre pour amener le patient à la transplantation en prévenant ou traitant les diverses complications, y compris avec les moyens de support extracorporels.

\section{Autres complications}

\section{Péritonite bactérienne spontanée}

Une ponction d'ascite doit être pratiquée systématiquement. Les critères diagnostics d'une péritonite bactérienne sont $>500$ éléments nucléés $/ \mathrm{mm}^{3}$ ou $>250 \mathrm{PMN} / \mathrm{mm}^{3}$. Le traitement peut être limité à de l'amoxycilline/clavulanate (Augmentin ${ }^{\circledR}$ ). 
Tableau VIII - Les indications de la transplantation hépatique dans l'insuffisance hépatique aiguë.

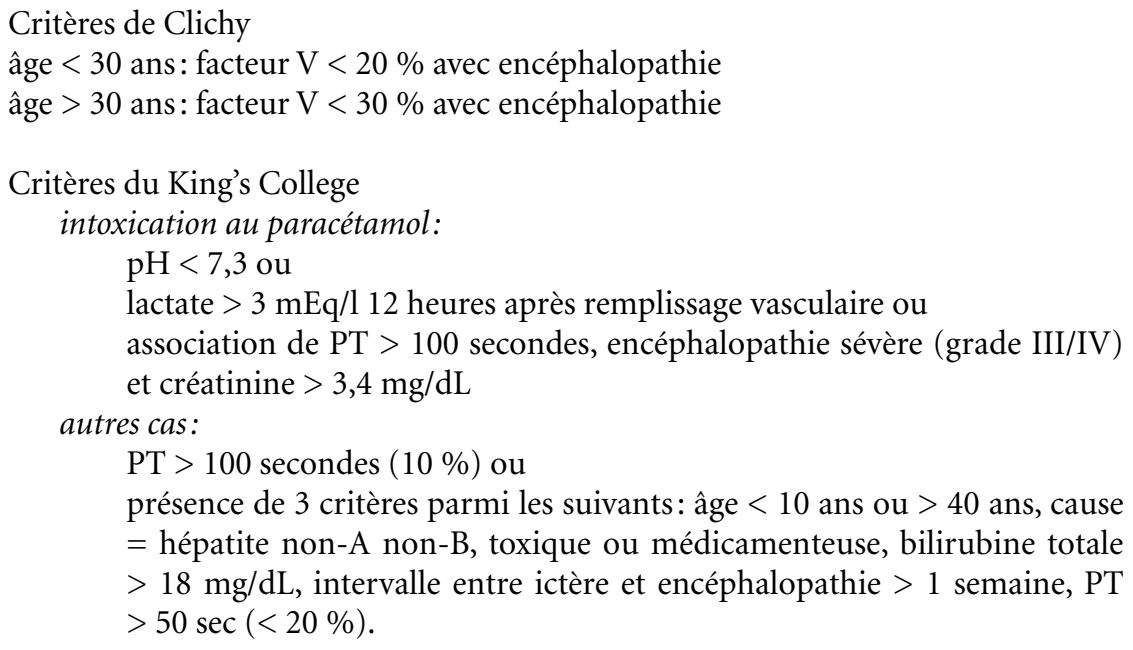

\section{Neurologiques}

L'encéphalopathie peut s'aggraver rapidement, et se compliquer alors d'œdème cérébral.

Attitude (voir plus haut: encéphalopathie hépatique):

- maintenir la tête légèrement surélevée à $30^{\circ}$ (une plus grande élévation risque de diminuer la perfusion cérébrale);

- éviter l'hyperventilation (qui risque d'entraîner une ischémie cérébrale) chez le malade ventilé mécaniquement et en encéphalopathie sévère, mais maintenir un certain degré d'hypocapnie (30-35);

Dans les cas les plus graves, certains mesurent la pression intracrânienne (cathéter extradural), avec le but de la maintenir en dessous de $30 \mathrm{mmHg}$;

- mannitol (solution à $20 \%$ ): 0,5 mg/kg à $1 \mathrm{~g} / \mathrm{kg}$ en 10 minutes, en s'assurant que l'osmolarité plasmatique n'excède pas $320 \mathrm{mOsm}$;

- éventuellement coma barbiturique (thiopental 3-5 mg/kg - voir chapitre Soins intensifs neurologiques).

\section{Respiratoires}

Il s'agit surtout d'infections ou ARDS (inhalation).

Le malade présente généralement une hyperventilation spontanée, si bien que la ventilation mécanique est plus souvent indiquée en raison de l'hypoxémie et/ou de l'altération de la conscience.

\section{Cardiovasculaires}

Le malade présente typiquement un tableau hyperkinétique (débit cardiaque élevé) comme au cours du sepsis. Au vu des anomalies d'extraction d'oxygène, il faut maintenir le débit cardiaque à des valeurs supranormales. L'administration de 
vasopresseurs (dopamine et même noradrénaline) est indiquée en cas d'hypotension artérielle résistante au «fluid challenge».

\section{Métaboliques}

L'hyponatrémie est fréquente: elle résulte de la rétention hydrique avec entrée de sodium dans la cellule suite à l'inhibition de la $\mathrm{Na} / \mathrm{K}$ ATPase membranaire. L'hypokaliémie et l'hypophosphorémie sont également fréquentes.

L'hypoglycémie peut survenir dans les états graves: il faut alors surveiller la glycémie au moins toutes les 4 heures, et perfuser des solutions de glucosé à 10 ou $20 \%$; éventuellement bolus de glucosé $30 \%$.

\section{Insuffisance rénale}

Le diagnostic différentiel entre syndrome hépato-rénal (insuffisance prérénale) et néphrite tubulo-interstitielle n'est pas évident.

Le syndrome hépato-rénal est une insuffisance rénale fonctionnelle, de type prérénal, avec augmentation de l'osmolarité urinaire et natriurèse faible (voir chapitre Insuffisance rénale). La fonction rénale peut être entièrement restaurée après transplantation hépatique.

La physiopathologie est complexe, mettant en jeu des anomalies de la fonction endothéliale, avec production accrue de prostanoides, d'oxyde nitrique et d'endothéline.

Les critères actuels sont présentés dans le tableau IX:

Tableau IX - Critères actuels du syndrome hépato-rénal.

Critères absolus

- Cirrhose

- créatininémie $>1,5 \mathrm{mg} / \mathrm{dL}$ ou clairance de créatinine $<40 \mathrm{~mL} / \mathrm{min}$

- absence d'infection, de choc ou de pertes liquidiennes importantes

- absence de traitement récent par agents néphrotoxiques

- pas d'amélioration après arrêt des diurétiques et la perfusion d'1,5 L de solution salée

- protéinurie $<500 \mathrm{mg} /$ jour

- pas d'évidence de pathologie rénale parenchymateuse ou obstructive.

Critères relatifs

- Diurèse $<500 \mathrm{~mL} /$ jour

- Natriurèse $<10 \mathrm{mEq} / \mathrm{L}$

- Uosm $>$ Posm

- GR unines $<50 /$ champ

- Na sanguin $<130 \mathrm{mEq} / \mathrm{L}$

\section{Attitude}

Il est essentiel de maintenir une volémie efficace suffisante.

En cas d'insuffisance rénale installée, l'hémodiafiltration est préférable à l'hémodialyse pour minimiser les shifts importants de solutés. 
L'administration de vasopressine semble utile. La terlipressine (Glypressine ${ }^{\circledR}$ ) est un analogue de la vasopressine, qui peut être administré sous forme de bolus intraveineux, car il est lentement activé dans la circulation. Elle est aussi efficace que la vasopressine, mais présente moins d'effets secondaires. La dose est de 0,5 mg toutes les 4 heures.

Les perfusions d'albumine $(0,5$ à $1 \mathrm{~g} / \mathrm{kg} /$ jour $)$ sont généralement associées pour maintenir une volémie efficace.

\section{Altérations de coagulation}

Elles sont responsables de saignements divers, en particulier digestifs.

Le déficit en facteurs 1, II, V, VII, IX et X est responsable de l'allongement du PT. Le facteur VII, dont la demi-vie est courte, diminue précocement. La chute du facteur $\mathrm{V}$ est spécifique de l'insuffisance hépatique aiguë, si bien que son dosage (en \% de la normale) est utile à évaluer la sévérité de l'insuffisance hépatique. À l'opposé, le facteur VIII est préservé. Du FFP ne doit être administré que s’il y a saignement; son administration systématique est inutile et à éviter, car elle rend impossible l'évaluation de la sévérité de l'insuffisance hépatique.

\section{Infectieuses}

L'infection apparaît dans la majorité des cas; l'infection fongique est relativement fréquente. Une ponction d'ascite doit systématiquement être réalisée en cas de sepsis. La présence de plus de 500 leucocytes ou de 250 neutrophiles $/ \mathrm{mm}^{3}$ nécessite une antibiothérapie, généralement sous forme d'une céphalosporine de $3^{\mathrm{e}}$ génération.

Certains préconisent la décontamination sélective du tube digestif (SDD).

Traitement additionnel

$\mathrm{N}$-acétylcystéine (augmente les réserves en glutathion): $300 \mathrm{mg} / \mathrm{kg}$ en 20 heures: surtout en cas d'intoxication au paracétamol, mais aussi parfois administré dans les autres cas.

Ponction d'ascite: une perfusion d'albumine est généralement associée pour maintenir la volémie.

Transplantation hépatique: (voir tableau VIII);

La survie à un an avoisine actuellement les $60 \%$.

En cas de nécrose hépatique responsable d'une insuffisance hépatique sévère, une hépatectomie complète est parfois réalisée, en espérant trouver un donneur en urgence endéans les 24-48 heures.

Systèmes extracorporels: système MARS ( molecular absorbent recirculating system ») ou Prometheus: il s'agit d'une dialyse utilisant un dialysat riche en albumine, pour faciliter l'élimination de toxines liées à l'albumine comme la bilirubine, les acides aminés aromatiques et des substances hydrosolubles. L'efficacité n'est pas encore définitivement prouvée.

Expérimental: foie artificiel extracorporel.

Mortalité: 80 \% sans transplantation hépatique.

Le pronostic est meilleur pour l'hépatite A et l'intoxication au paracétamol, intermédiaire pour l'hépatite B et le moins bon pour les autres intoxications ou les causes inconnues. Le degré d'encéphalopathie a une valeur pronostique incontestable.

La survie à un an après transplantation avoisine actuellement les 60 \%. 


\section{Altération des tests hépatiques - cholestase}

Ictère à bilirubine conjuguée ou non conjuguée

Rappelons que la prédominance d'hyperbilirubinémie non conjuguée doit faire suspecter une hémolyse. L'hyperbilirubinémie conjuguée (> $50 \%$ ) non expliquée suggère avant tout le sepsis (fig. 3 ).

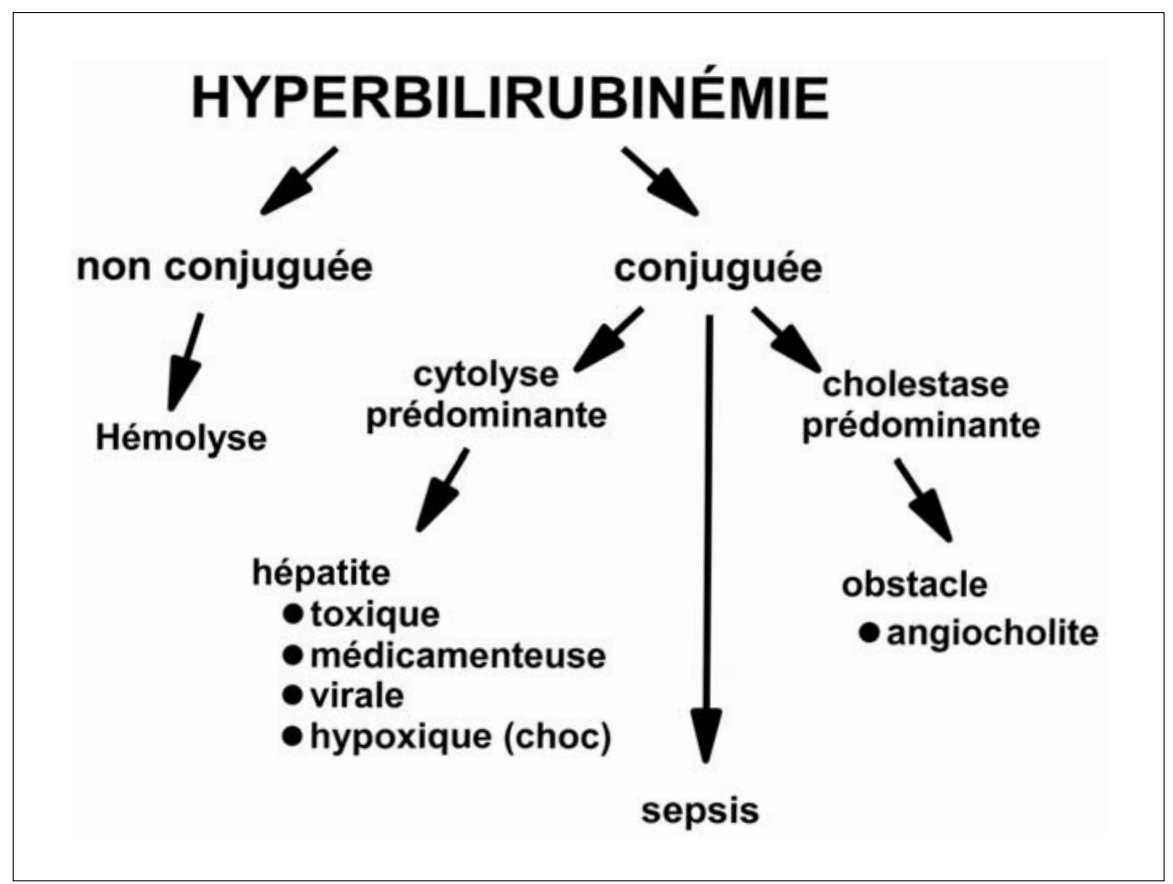

Fig. 3 - Les hyperbilirubinémies.

Cholécystite et cholangite

L'obstruction des voies biliaires est un diagnostic à exclure en présence de fièvre d'origine indéterminée ou d'hyperbilirubinémie mal expliquée.

\section{Cholécystite alithiasique}

Elle représente une minorité de cas de cholécystite. Elle survient plus souvent chez les hommes, et en général après un séjour prolongé en soins intensifs, et en général chez les patients chirurgicaux.

L'élément important ici est l'ischémie vésiculaire, favorisée par l'hypovolémie et sans doute d'autres facteurs comme les cytokines, la ventilation mécanique, l'alimentation parentérale, peut-être les opiacés (augmentation de la pression au niveau du sphincter d'Oddi... 
Les signes cliniques fréquents sont la douleur dans l'hypochondre droit et la douleur à la palpation à ce niveau. La mise en évidence se fait généralement par échographie et/ou par CT-scan abdominal. L'examen échographique montre un épaississement (3 à 3,5 mm) de la paroi vésiculaire, avec parfois présence de liquide extravésiculaire. Le diagnostic est essentiel, car l'ischémie vésiculaire peut évoluer vers la gangrène et la perforation.

Le traitement consiste en une cholécystectomie chirurgicale ou percutanée (éventuellement sous anesthésie locale).

\section{Rôle des médicaments}

L'altération des tests hépatiques est parfois due aux médicaments (antiépileptiques, kétoconazole, INH, paracétamol...).

\section{Rôle de l'alimentation parentérale}

L'alimentation parentérale en elle-même n'est pas hépatotoxique, mais une augmentation des transaminases ( 5 à 10 fois la valeur normale) peut survenir en présence d'excès caloriques $(50 \mathrm{Cal} / \mathrm{kg} / \mathrm{j})$ et rapidement résolutifs à l'arrêt de l'alimentation. On peut aussi observer une augmentation des taux de phosphatase alcaline, mais le phénomène est plus lent, de moindre magnitude, et davantage persistant à l'arrêt de l'alimentation parentérale.

Plusieurs causes peuvent être identifiées, y compris l'excès de glucose (oxydation maximale chez l'homme 5 à $7 \mathrm{mg} / \mathrm{kg} / \mathrm{min}$ ), l'excès de lipides, l'excès de calories quelle qu'en soit la nature, le manque de stimulation de la production de bile par le bol alimentaire avec stase biliaire, l'arrêt de la recirculation entéro-hépatique des sels biliaires, la déficience en oligoéléments conduisant à la stéatose.

\section{Monitorage de la région hépato-splanchnique}

La muqueuse gastrique ou intestinale est un endroit particulièrement sensible pour plusieurs raisons:

- le débit régional hépato-splanchnique est diminué précocément au cours des insuffisances circulatoires pour tenter de préserver le débit régional aux organes vitaux (le tube digestif n'est pas un organe prioritaire au cours des épisodes de stress);

- la muqueuse digestive a une architecture microvasculaire particulière caractérisée par le passage d'oxygène à contre-courant et un hématocrite du sang capillaire plus bas que dans d'autres tissus, expliquant le risque important d'ischémie à l'extrémité des villosités;

- le tube digestif est riche en micro-organismes et l'ischémie régionale pourrait favoriser le passage de ces produits (notamment les endotoxines) dans la circulation (translocation).

\section{Tonométrie gastrique}

Le principe de base est que la pression partielle en $\mathrm{CO}_{2}\left(\mathrm{PCO}_{2}\right)$ est identique dans la lumière d'un organe et dans la muqueuse qui l'entoure, grâce à la grande solubilité 
du $\mathrm{CO}_{2}$. Le placement d'un ballonnet perméable au $\mathrm{CO}_{2}$ permet de doser la $\mathrm{PCO}_{2}$ du liquide salé ou du gaz placé à l'intérieur du ballonnet.

Le $\mathrm{pH}$ intramuqueux gastrique $(\mathrm{pHi})$ peut être calculé par l'équation de Henderson-Hasselbalch, à partir de la $\mathrm{PCO}_{2}$ gastrique $\left(\mathrm{PgCO}_{2}\right)$ et du bicarbonate artériel. Le calcul du pHi est aujourd'hui évité, en raison de ses problèmes:

- en cas d'ischémie régionale (mésentérique), le bicarbonate régional peut être plus bas que celui mesuré dans une artère périphérique, si bien que le pHi sera surestimé;

- une diminution du bicarbonate artériel (acidose métabolique) peut être due à d'autres facteurs (insuffisance rénale, acido-cétose, etc.) qui n'impliquent pas une diminution de perfusion du tube digestif.

Tableau X - Causes d'augmentation de $\mathrm{PCO}_{2}$ régionale $\left(\mathrm{PgCO}_{2}\right)$.

Une diminution du débit cardiaque sanguin total (le gradient entre $\mathrm{PvCO}_{2}$ et $\mathrm{PaCO}_{2}$ sera aussi élargi).

Une diminution du débit sanguin de la muqueuse gastrique.

Une hypoxie (dysoxie) régionale avec développement de métabolisme anaérobie et production accrue d'ions $\mathrm{H}+$.

Une hypoxie cytotoxique (au cours du sepsis).

Une augmentation de la $\mathrm{PaCO}_{2}$ (le gradient entre $\mathrm{PgCO}_{2}$ et $\mathrm{PaCO}_{2}$ sera alors normal).

Tableau XI - Éléments bien établis et moins bien établis à propos de la tonométrie gastrique.

Éléments bien établis:

- le pHi est souvent abaissé dans les affections aiguës, même en l'absence de choc circulatoire;

- un pHi abaissé est associé à un moins bon pronostic, surtout lorsque l'anomalie persiste;

- les effets d'interventions thérapeutiques sur le pHi sont loin d'être prévisibles et reproductibles;

- il est préférable de discuter le $\mathrm{PCO}_{2}$ gap plutôt que le pHi ou le pHgap

Éléments moins bien établis:

- le pronostic du $\mathrm{PCO}_{2}$ gap élevé (plutôt que du pHi abaissé) n'est pas bien établi;

- le degré de nécessité de l'administration d'anti- $\mathrm{H}_{2}$ et d'absence d'alimentation entérale;

- un $\mathrm{PCO}_{2}$ gap élevé peut refléter d'autres phénomènes qu'un apport en oxygène insuffisant à la muqueuse gastrique;

- on ne peut recommander aujourd'hui de guider les interventions thérapeutiques par le $\mathrm{PCO}_{2}$ gap. 
La tonométrie en phase gazeuse (ou la mesure de la $\mathrm{PCO}_{2}$ par senseur fibroptique) est préférée, pour avoir une mesure quasi continue plutôt qu'intermittente, et éviter les erreurs de manipulations et les erreurs de mesure d'analyse de gaz de la solution salée.

$\mathrm{La} \mathrm{PgCO}_{2}$ étant directement influencée par la $\mathrm{PaCO}_{2}$, il faut toutefois calculer le gradient entre $\mathrm{PgCO}_{2}$ et $\mathrm{PaCO}_{2}$; ce « $\mathrm{PCO}_{2}$ gap » est normalement inférieur à $6 \mathrm{mmHg}$.

La mesure est influencée par l'acidité de l'estomac, si bien que l'administration systématique d'anti- $\mathrm{H}_{2}$ est probablement souhaitable. De même, la nutrition peut réduire le $\mathrm{pH}$ gastrique, si bien que les mesures devraient être effectuées 1 heure après l'interruption de la nutrition entérale. Mieux encore, le $\mathrm{pH}$ gastrique devrait être mesuré régulièrement, pour s'assurer qu'il n'est pas acide.

Les interventions thérapeutiques les plus susceptibles de diminuer le $\mathrm{PCO}_{2}$ gap (augmenter le $\mathrm{pHi}$ ) sont le «fluid challenge» et l'administration de dobutamine. Les résultats concernant la dopexamine et la noradrénaline sont plus controversés. La dopamine et l'adrénaline tendent à accroître le $\mathrm{PCO}_{2}$ gap. Toutefois, l'effet de ces interventions thérapeutiques est loin d'être prévisible.

Une série d'études cliniques ont clairement établi une relation entre un pHi bas $(<7,32)$ et un moins bon plus pronostic. Cette relation est moins évidente pour le $\mathrm{PCO}_{2}$ gap (quand la valeur pronostique du bicarbonate artériel est retirée). D'autres investigations sont nécessaires avant d'éventuellement proposer ce type de monitorage en routine chez le malade grave.

Mesure de la circulation muqueuse gastrique

L'utilisation d'un débitmètre laser-Doppler attaché à une sonde gastrique permet d'apprécier la perfusion muqueuse régionale (à l'endroit où la sonde est appliquée), mais l'information est relative plutôt qu'absolue et est susceptible d'être influencée par le degré de pression de l'appareil sur la muqueuse.

\section{Mesure de l'oxygénation muqueuse}

L'appareil «Erlanger Micro-lightguide Photometer » (EMPHO) utilise une technique de spectrophotométrie pour déterminer le contenu relatif en oxyhémoglobine.

Mesure du débit sanguin hépato-splanchnique et de l'extraction d'oxygène au niveau hépatique

Le placement d'un cathéter sus-hépatique (sous contrôle fluoroscopique) permet un accès à la circulation veineuse sus-hépatique.

La mesure la plus fiable du débit sanguin hépatique est la mesure de clairance de vert d'indocyanine, pour autant que l'analyse se fasse au niveau régional (le degré d'extraction de vert d'indocyanine par d'autres tissus est variable).

La méthode recommandée est celle de la perfusion continue («steady state»), en appliquant la formule:

IRICG

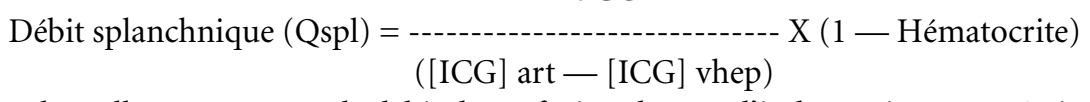

dans laquelle IR représente le débit de perfusion du vert d'indocyanine en $\mathrm{mg} / \mathrm{min}$. 
La mise en place d'un cathéter sus-hépatique permet aussi d'y mesurer la saturation en oxygène du sang veineux $\left(\mathrm{ShO}_{2}\right)$. Chez la malade en état critique (surtout septique), l'extraction d'oxygène au niveau hépato-splanchnique est un peu plus importante qu'au niveau global, si bien que la $\mathrm{ShO}_{2}$ est un plus basse que la $\mathrm{SvO}_{2}$ : sa valeur normale est aux environs de $65 \%$. Une $\mathrm{ShO}_{2}$ plus basse suggère la présence d'une inadéquation des apports par rapport aux besoins en oxygène dans cette région.

\section{Mesure du métabolisme de la lidocaïne}

Ce test consiste en l'administration de $1 \mathrm{mg} / \mathrm{kg}$ de lidocaïne dont on mesure la concentration en métabolite, le monoethylglycinexylidide (MEGX) après exactement 15 min; chez l'individu normal, la concentration est de 75-100 $\mu \mathrm{g} / \mathrm{L}$, tandis qu'une concentration $<25 \mu \mathrm{g} / \mathrm{L}$ suggère fortement une dysfonction hépatique, encore que le test soit influencé par le débit sanguin hépatique.

\section{Mesure de la perméabilité intestinale}

Elle se fait essentiellement par administration simultanée de saccharides de poids moléculaire différent pour pouvoir établir le rapport d'élimination (ex.: rhamnose/lactulose) ou du 51Cr-EDTA pour apprécier le degré de perméabilité du tube digestif. Le problème est la grande sensibilité de ces tests (déjà altérés par exemple après circulation extracorporelle), si bien que leur utilité clinique n'est pas établie.

\section{Pancréatites}

\section{Physiopathologie}

Le pancréas contient une série de substances protéolytiques et lipolytiques qui pourraient entraîner des dégâts considérables dans l'organisme. L'autodigestion est prévenue par trois mécanismes essentiels:

- les enzymes sont libérés sous forme inactive (comme le trypsinogène) ;

- les enzymes sont gardés dans des systèmes de réserve dans la cellule;

- il existe des inhibiteurs des protéases, comme l'alpha-1 antiprotéase ou l'alpha-

2 macroglobuline.

Bien que la physiopathologie de la pancréatite ne soit pas encore bien précisée, on incrimine la libération d'enzymes dans la cellule glandulaire, aboutissant à une autodigestion de la glande et des tissus adjacents par la trypsine et la libération de protéases. La pancréatite devient un phénomène inflammatoire sévère, qui implique notamment

- l'activation du complément ;

- les cytokines pro-inflammatoires (TNF, l'IL-1, l'IL-6 et l'IL-8...) ;

- le système kinine-kallikreine, qui participe à la vasodilatation, l'augmentation de perméabilité vasculaire, l'activation leucocytaire;

- la chémotrypsine, qui participe à la libération de radicaux libres d'oxygène;

- l'elastase, qui participe aux lésions vasculaires;

- la lipase, qui entraîne la nécrose graisseuse; 
- la phospholipase A2, qui altère directement les membranes cellulaires;

- les radicaux libres oxygène;

- le «platelet activating factor» (PAF).

Bien que la plupart des pancréatites soient modérées et non compliquées, environ $20 \%$ sont sévères, aboutissant à un tableau de pancréatite nécrosante. Une pancréatite sévère peut entraîner un tableau de MOF (ARDS, insuffisance rénale, altérations de coagulation, altération de l'état mental...).

Plus des 2/3 des cas sont dus à une pathologie des voies biliaires ou l'éthylisme. Les autres causes sont diverses chez le malade grave: trauma, états postopératoires compliqués, choc, sepsis, virus, circulation extracorporelle, médications diverses...

Pour tout épisode de pancréatite, il faut donc préciser:

- une cause possible;

- s'il s'agit d'un épisode aigu unique ou d'une affection récurrente;

- le degré de sévérité des lésions pancréatiques;

- le degré de répercussion sur les autres organes.

\section{Diagnostic et évaluation}

Le choc circulatoire est présent dans les cas les plus sévères. La fièvre peut être présente, et ne signe pas nécessairement la présence d'infection. L'examen montre souvent une moindre dépression de la région épigastrique. Le péristaltisme peut être diminué ou absent, et le tableau clinique peut être celui d'abdomen aigu. Une coloration bleuâtre des flancs (signe de Grey-Turner) ou de la région périombilicale (signe de Cullen) peut être parfois observée dans des cas graves: ces signes reflètent la présence d'hémorragie rétropéritonéale.

Examens biologiques

Amylase: le diagnostic de pancréatite est souvent suggéré par la découverte d'une hyperamylasémie. Il faut toutefois se rappeler que l'hyperamylasémie est peu spécifique: elle peut s'élever dans l'ulcère duodénal perforé, l'infarctus mésentérique, l'obstruction intestinale, etc. On se rappelle également que la perfusion d'HES peut augmenter l'amylase sans pancréatite. De plus, l'insuffisance rénale peut contribuer à l'hyperamylasémie. À l'inverse, les taux d'amylase peuvent parfois être normaux dans une pancréatite très sévère.

Lipase: plus spécifique; toutefois l'insuffisance rénale peut aussi contribuer à son élévation.

Examen hématologique: une hyperleucocytose et une anémie sont souvent présentes, mais évidemment non spécifiques.

Hyperglycémie: souvent présente (non seulement liée à la diminution de libération d'insuline mais aussi à la libération accrue de glucagon, de glucocorticoïdes et de catécholamines) : peut nécessiter l'administration d'insuline.

Protéines de la phase aiguë: surtout la CRP (surtout dans les cas graves).

Calcium ionisé: l'hypocalcémie est fréquente et doit être traitée en cas de choc circulatoire.

Radiographie de l'abdomen à blanc: peu utile, sinon pour exclure d'autres diagnostics (organe perforé); la radio peut visualiser les calcifications pancréatiques ou des signes non spécifiques, comme l’anse sentinelle. 
Échographie: est généralement peu contributive en ce qui concerne le pancréas, qui n'est visualisé dans moins de la moitié des cas, surtout si un iléus est responsable d'une dilatation des anses intestinales. L'examen est surtout utile dans deux cas:

- pour déceler une pathologie biliaire;

- pour évaluer la progression de pseudo-kystes.

CT-scan abdominal: c'est l'examen de choix pour :

- établir la présence de pancréatite (et exclure d'autres diagnostics comme un infarctus mésentérique ou un ulcère perforé);

- établir le degré des lésions; l'œdème pancréatique sans extension extérieure est évidemment de moindre gravité et de meilleur pronostic que la pancréatite nécrosante ;

- détecter les complications locales et éventuellement guider l'aspiration et le drainage de la nécrose ou de collections liquidiennes.

L'examen doit être répété pour surveiller l'évolution du processus.

La sévérité est évaluée par le degré d'élargissement du pancréas, la présence d'inflammation et la présence de collections liquidiennes ainsi que leur localisation. La classification de Balthazar est souvent utilisée (tableau XII).

L'injection de produit de contraste avec phase artérielle précoce est indispensable pour distinguer les lésions interstitielles des lésions nécrotiques et apprécier la sévérité. Les lésions interstitielles ont une microcirculation intacte et une augmentation globale de la captation, tandis que les lésions nécrotiques entraînent une interruption de la microcirculation sans captation du produit.

Tableau XII - Classification de Balthazar.

Grade A: normal

Grade B: élargissement local ou diffus du pancréas;

Grade C: id + inflammation péri pancréatique modérée;

Grade D: id + collection liquidienne unique (généralement dans l'espace antérieur, pararénal);

Grade E: id + liquide dans deux régions au moins et/ou présence de gaz dans la région pancréatique.

\section{Résonance magnétique nucléaire (RMN)}

Sa supériorité par rapport au CT-scan n'est pas encore clairement établie, si bien que cet examen, plus difficile à réaliser chez le malade grave, n'est pas recommandé aujourd'hui. Elle peut cependant apporter des précisions importantes sur les morphologies canalaires (rupture du wirsung, fistules internes). 


\section{Évaluation de la sévérité}

Le degré de sévérité de la pancréatite peut être évalué à l'aide des critères de Ranson (voir tableau XIII), qui sont cependant moins utilisés aujourd'hui. Les scores de sévérité non spécifiques (APACHE, SAPS) ou le degré de dysfonction des organes (score SOFA) quantifient aussi bien la sévérité de la pancréatite.

\section{Traitement}

La réanimation initiale de la pancréatite repose sur la règle du VIP. Il faut souligner que des quantités parfois très importantes de perfusions peuvent être nécessaires, dans la mesure où les pertes liquidiennes peuvent être très importantes. La libération importante de médiateurs entraîne aussi un désordre de type distributif.

\section{Support nutritionnel}

La mise au repos du tube digestif était systématique dans le passé, mais aucune étude n'a démontré l'avantage de cette stratégie. L'arrêt de l'alimentation entérale est réservé aux cas les plus graves. L'aspiration gastrique est utile en cas de vomissements importants, mais ne doit pas nécessairement être appliquée en routine. Même la mise en place en routine d'une sonde gastrique n'est plus recommandée aujourd'hui dans les cas peu sévères, car il n'y pas d'évidence que l'aspiration gastrique améliore le pronostic.

L'alimentation entérale peut être poursuivie par voie jéjunale. Bien que le bénéfice n'est pas définitivement démontré, cette stratégie est au moins efficace et non délétère.

Tableau XIII - Critères de Ranson.

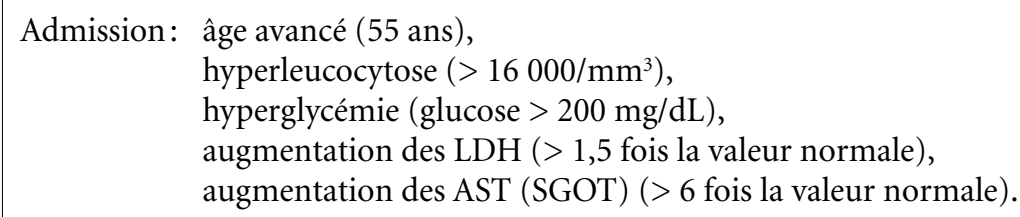

Endéans les 48 heures: chute d'hématocrite (>10\%), augmentation de l'urée (de $10 \mathrm{mg} / \mathrm{dL}$ )

hypocalcémie (calcium $<8 \mathrm{mg} / \mathrm{dL}$ ), hypoxémie $\left(\mathrm{PaO}_{2}<60 \mathrm{mmHg}\right.$ à l'air ambiant $)$, acidose métabolique (base déficit $>4 \mathrm{mEq} / \mathrm{L}$ ), balance liquidienne positive de $>6$ litres.

$>3 / 11$ critères présents $=$ pancréatite sévère 
En cas de mise au repos du tube digestif, une alimentation parentérale peut être requise. L'administration de graisses n'est pas contre-indiquée, sauf en cas d'hypertriglyceridémie importante (> $500 \mathrm{mg} / \mathrm{dL}$ ).

\section{Antibiotiques}

Bien que la place exacte du traitement antibiotique soit encore débattue, on tend à systématiquement administrer des antibiotiques dans les cas graves, car la translocation bactérienne à partir du tube digestif pourrait jouer un rôle dans les infections secondaires. On préfère un antibiotique à large spectre (car les germes incriminés sont multiples) et qui pénètre suffisamment bien dans le pancréas. Le choix se porte dans l'ordre sur une peneme (imipenem ou meropenem), une quinolone (ciprofloxacine ou ofloxacine) ou une céphalosporine de $3^{\text {e }}$ génération (céfotaxime ou ceftriaxone).

Évidemment, un traitement antibiotique est indiqué en cas de nécrose infectée.

Drainage ou autres procédures invasives

Une ERCP avec papillotomie doit être pratiquée en cas d'obstruction des voies biliaires par un calcul ou en présence d'angiocholite. La procédure est faisable sans grande complications et est à pratiquer en urgence ( $24 \mathrm{~h}$ de l'admission).

La cholécystectomie (éventuellement par cœlioscopie) est nécessaire en cas de lithiases biliaires, mais le moment idéal est souvent difficile à définir.

La chirurgie a une place limitée dans les pancréatites nécrosantes (et n'a aucune place dans la pancréatite œdémateuse). La chirurgie précoce " de nettoyage » a des effets défavorables et doit être à tout prix évitée. Par contre, il est essentiel de drainer les collections infectées (nécrose infectée ou abcès) sans délai par voie chirurgicale, endoscopique ou transcutanée (sous contrôle de CT-scan). Le problème est que l'infection est présente dans environ la moitié des cas, et le diagnostic est difficile. Les signes cliniques de sepsis sont peu utiles, étant donné qu'ils peuvent être présents en l'absence comme en présence d'infection.

L'infection peut être révélée par:

- la présence de micro-organismes lors de l'aspiration de liquide à l'aiguille fine, sous contrôle CT -scan;

- l'élévation prolongée de taux de CRP ou de procalcitonine;

- une bactérémie;

- la présence de gaz au CT -scan.

Les pseudo-kystes éventuels ne doivent pas être drainés, sauf s'ils deviennent symptomatiques (infection ou douleurs persistantes).

\section{Antidouleurs}

Les morphiniques peuvent créer un spasme du sphincter d'Oddi, mais ne sont pas pour autant contre-indiqués.

Antiémétiques éventuels.

Traitement médicamenteux d'utilité non prouvée:

- inhibiteurs de protéases: aprotinine (Trasylol ${ }^{\circledR}$ ) ou nafamostat: pas d'évidence; peut-être parce que ces substances ne peuvent pénétrer dans la glande pour y 
exercer leurs effets. Le mesylate de gabexate, avec un poids moléculaire plus bas, et qui inhibe aussi la phospholipase A2, pourrait être plus efficace (mais aussi non prouvé);

- FFP pour apporter des inhibiteurs des protéases;

- Plasmaphérèse: dans le but d'éliminer une série de médiateurs;

- Atropine: réduit la pression du sphincter d'Oddi, réduit la sécrétion gastrique et donc la stimulation pancréatique médiée par la sécrétine;

- glucagon: inhibiteur du pancréas exocrine;

- somatostatine ou octréotide (Sandostatine ${ }^{\circledR}$ ) pour inhiber la sécrétion exocrine: pas d'évidence solide, puisque la sécrétion pancréatique est plutôt diminuée. De plus, ces substances diminuent le débit sanguin pancréatique.

- lavage péritonéal basé sur le principe que la cavité péritonéale est un réservoir d'enzymes pancréatiques. Il en va de même du drainage du canal lymphatique thoracique, préconisé dans le même but.

- antioxydants comme la N-acétylcystéine;

- antagonistes du « platelet activating factor» (PAF), comme le lexipafant. 